\title{
Collembolan grazing affects the growth strategy of the cord-forming fungus Hypholoma fasciculare
}

\author{
Christian Kampichler ${ }^{\mathrm{a}, *}$, Johann Rolschewski ${ }^{\mathrm{a}}$, Damian P. Donnelly ${ }^{\mathrm{b}}$, Lynne Boddy ${ }^{\mathrm{b}}$ \\ ${ }^{\mathrm{a}}$ Soil Zoology and Ecology Laboratory, Freie Universität Berlin, Grunewaldstrasse 34, D-12165 Berlin, Germany \\ ${ }^{\mathrm{b}}$ Cardiff School of Biosciences, Cardiff University, P.O. Box 915, Cardiff CF10 3TL, UK
}

Received 11 June 2003; received in revised form 20 November 2003; accepted 17 December 2003

\begin{abstract}
Mycelia of cord-forming fungi show remarkable patterns of reallocation of biomass and nutrients indicating an important role of these, often extensive, organisms in the spatial translocation of energy and nutrients in forest soils. Despite the rich tradition of interaction studies between soil microarthropods and fungi, the spatial implications of these interactions, due to the potential growth responses of the fungi and to the translocation of energy and nutrients within the mycelial network, have been largely ignored. In this paper we analyse fungal growth responses in two-dimensional model systems composed of compressed soil, the cord-forming fungus Hypholoma fasciculare and three fungivorous Collembolan species. We hypothesised that (i) the highly co-ordinated nature of cord-forming fungi would lead to growth responses following collembolan grazing, and that, (ii) such changes are dependent on grazing intensity, and (iii) changes are dependent on the species grazing. Mycelial extent and hyphal cover decreased with increasing grazing density; at highest grazing density also the fractal dimension of the mycelial border decreased, indicating a less branched foraging front due to the regression of fine hyphae and the development of mycelial cords. Effects differed greatly between collembolan species although they exerted comparable grazing pressure (the smaller species were added in larger numbers according to their allometric size-metabolic rate relationships): while grazing by Folsomia candida resulted in less mycelial extension and hyphal cover, these variables were not affected when Proisotoma minuta and Hypogastrura $c f$. tullbergi grazed. The effects of a species mix suggested an additive effect of the component species. This shows that fungal mycelia may suffer from damage caused by few but large collembolans, affecting extension as well as coverage of the mycelium, but that fungi may compensate for the biomass loss caused by more but slightly smaller collembolans. In about $20 \%$ of the model systems $\mathrm{H}$. fasciculare switched from a growth pattern with a broad contiguous foraging front and uniform growth in all directions to a pattern with fast growing sectors while other sectors stopped growth completely. The switch occurred in grazed systems exclusively; thus we interpret this observation as a fugitive response and as a strategy for quickly escaping from places where grazing pressure is experienced.
\end{abstract}

(C) 2004 Published by Elsevier Ltd.

Keywords: Hypholoma fasciculare; Cord-forming fungi; Collembola; Grazing; Microarthropod-microbial interactions; Growth strategies; Fractal dimension; Mycelial extent; Hyphal cover

\section{Introduction}

Saprotrophic cord-forming fungi are complex organisms showing a wide range of behaviour patterns when foraging for resources (Boddy, 1999). They are 'non-resource-unitrestricted' which means that while 'resource-unit-restricted' fungi are confined to their organic resource and need to

\footnotetext{
* Corresponding author. Present address: Academic Division of Biological Sciences, Universidad Juárez Autónoma de Tabasco, Carretera Villahermosa-Cárdenas Km. 0.5 S/N, Entronque a Bosques de Saloya, 86150 Villahermosa, Tabasco, Mexico. Tel.: +52-44993-3065148; fax: +49-1212-5250-27314/+52-993-3544308.

E-mail address: christian.kampichler@web.de (C. Kampichler).
}

disseminate to new resources by spores, nonresource unit restricted fungi are able to extend from a resource as mycelium and may thus physically interconnect discrete organic resources. These mycelial systems can reach a size of more than $100 \mathrm{~m}$ diameter (Boddy, 1993) and, consequentally, we find among these fungi some of the oldest and largest living organisms on earth (Smith et al., 1992). Cord-forming fungi characteristically produce linear organs consisting of aggregations of predominantly parallel, longitudinally aligned hyphae behind a diffuse mycelial growth front (Thompson and Rayner, 1982; Boddy, 1993). Cordformers are abundant on the floor of tropical, temperate and boreal forests, typically extending between discrete woody 
resources, and can even colonise tropical forest canopies (Boddy, 1999). Cord-former foraging strategies have been extensively studied along with responses to various environmental parameters, e.g. soil nutrient effects (Boddy et al., 1999), microclimatic effects (Donnelly and Boddy, 1997), responses to encounter with new resources (Wells et al., 1998) and responses to encounter with competing fungi (Boddy, 2000). In these studies the mycelia of cordforming fungi showed remarkable patterns of reallocation of biomass and nutrients (Boddy, 1999) indicating an important role of these, often extensive, organisms in the spatial translocation of energy and nutrients in forest soils.

Despite many previous studies on interaction between soil microarthropods and fungi (for example, van der Drift and Jansen, 1977; Booth and Anderson, 1979; Bengtsson and Rundgren, 1983; Moore et al., 1985; Verhoef and de Goede, 1985; Leonard and Anderson, 1991; Varga et al., 2002), the spatial implications of these interactions, due to the potential growth responses of the fungi and to the translocation of energy and nutrients within the mycelial network, have been largely ignored. We are aware of only a single paper on this topic: Hedlund et al. (1991) showed that grazing by a Collembolan induced a switch in mycelial morphology of the fungus Morteriella isabellina in agar culture. Interestingly, the grazing location and subsequent locations of mycelial responses were not identical, indicating a propagation of information through the mycelium. This paper analyses of fungal growth responses in a more realistic setting, in 2-dimensional model systems composed of compressed soil, the cord-forming fungus Hypholoma fasciculare and three fungivorous Collembolan species. We hypothesise that (i) the highly co-ordinated nature of cordforming fungi will lead to growth responses following collembolan grazing, and that, (ii) such changes are dependent on grazing intensity, and (iii) changes are dependent on which species is grazing.

\section{Materials and methods}

\subsection{Fungal and collembolan cultures}

H. fasciculare is a common and widespread wooddecomposing basidiomycete species colonising tree-stumps, dead roots, fallen branches and leaf litter. Originally isolated from tissue of a fruit body on a beech (Fagus sylvatica) $\log$, cultures were maintained and routinely subcultured on $2 \%(\mathrm{w} / \mathrm{v})$ malt extract agar. Beech wood inocula were prepared by putting $1 \mathrm{~cm}^{3}$ blocks into large jars (2 l) containing $30 \mathrm{~d}$ old $H$. fasciculare cultures on $500 \mathrm{ml}$ of malt extract agar and incubating at $20{ }^{\circ} \mathrm{C}$ in darkness for 10 weeks.

Collembolans of the species Folsomia candida, Proisotoma minuta and Hypogastrura cf. tullbergi were reared on a plaster of Paris:charcoal (4:1) substrate. They were provided weekly with yeast (Saccharomyces cerevisiae) and with pieces of bark of Platanus $\mathrm{x}$ hispanica covered with green algae. Eggs were transfered to new culture jars, thus all animals in a culture were of more or less the same age. Two days prior to the introduction of Collembola into the experimental systems, they were transfered to jars without food.

\subsection{Model systems}

We collected soil from the A-horizon ( $\mathrm{pH} 4.2$, maximum water capacity $16.0 \%$ of soil d.w., organic matter $2.3 \%$ of soil d.m.) of a beech stand near Chorin (Brandenburg, Germany). The unsterile loam was sieved (4 mm) and air-dried for 28 days. The soil was frozen $\left(-18{ }^{\circ} \mathrm{C}\right)$ for $24 \mathrm{~h}$ prior to experiments to kill any potential contaminant fauna, remoistened $(100 \mathrm{~g}$ air dried soil with $13 \mathrm{ml}$ distilled water) and $100 \mathrm{~g}$ of rewetted soil compressed into $14 \mathrm{~cm}$ dia. Petri dishes. Dishes were inoculated centrally with colonised beech wood blocks, and incubated at $18{ }^{\circ} \mathrm{C}$ in black plastic bags.

\subsection{Grazing intensity experiment}

We established four different grazing intensity levels (11 replicates each) with $0,20,40$ and 60 individuals of $F$. candida per tray. Collembolans were added 16 days after placement of the central wood inoculum, by which time the outgrowing mycelium had reached a radial extent of $1.5 \mathrm{~cm}$. The experiment was stopped when the mycelium reached the rim of the tray in one of the treatments.

\subsection{Response to different collembolan species}

Five different treatments were established (nine replicates each): $F$. candida only, $P$. minuta only, H. tullbergi only, a mix of all species and no Collembola (control) grazing. In order not to confound species effects with different grazing intensities we adjusted numbers of Collembola to their body size, thus ensuring comparable metabolic demands in all treatments except the control. Body lengths of Collembolan species (mean and standard deviation) were $1.96[0.19] \mathrm{mm}$ for $F$. candida, 1.35 [0.09] mm for $P$. minuta and 1.33 [0.09] $\mathrm{mm}$ for H. tullbergi. We estimated body mass by the relationship by Edwards (1967):

body mass $[\mu \mathrm{g}]=(C \times \text { body length }[\mathrm{mm}])^{3}$

where the constant $C$ is 3.06 for the species of the family Isotomidae ( $F$. candida, $P$. minuta) and 2.81 for the species of the family Hypogastruridae (H. tullbergi). Since in Collembola respiration rate scales as

respiration rate $\left[\mathrm{mm}^{3} \mathrm{O}_{2}\right.$ ind $\left.^{-1} \mathrm{~h}^{-1}\right]$

$=29.0 \times$ body mass $[\mu \mathrm{g}] 0.813$ 
(Dunger and Fiedler, 1997), the respiration rates of the three species are 9.78 ( $F$. candida), 3.94 ( $P$. minuta) and 4.64 ( $H$. tullbergi), corresponding to ratios of approximately 2.5:1 (F. candida:P. minuta) and 2:1 (F. candida:H. tullbergi). We thus placed 20 individuals of $F$. candida, 50 of $P$. minuta and 40 of $H$. tullbergi in the single-species treatments and seven individuals of $F$. candida, 17 of $P$. minuta and 13 of $H$. tullbergi in the mixed-species treatment. Thus, the grazing intensity in each treatment corresponded to the treatment with $20 \mathrm{~F}$. candida in the grazing intensity experiment.

As in the grazing intensity experiment, Collembolans were added when the outgrowing mycelium reached a radial extent of $1.5 \mathrm{~cm}$ (day 12), and the experiment was stopped when the mycelium reached the rim of the tray in one of the treatments.

\subsection{Image processing}

The experimental systems were photographed every 4$5 \mathrm{~d}$ with a Nikon Coolpix 990 digital camera from a distance of $40 \mathrm{~cm}$ and saved as images of size $512 \times 512$ pixels. We used SEMPER 9 (Synoptics, Cambridge, UK) for preprocessing the captured images which included high pass filtering (subtracting of the mean grey value averaged over the surrounding $120 \times 120$ pixel square, thus reducing any soil unevenness on the image), sharpening (doubling of pixel values and subtracting of the mean grey value of the suroounding $9 \times 9$ pixel square, thus enhancing the contrast between light and dark areas), histogram equalisation (rescaling of pixel values over range 0-255), windowing (manually removing inocula and the edge of the Petri dishes from the images), thresholding (converting pixels higher than the threshold to 255 , white, representing mycelium, and converting pixels lower than the threshold to 0, black, representing soil) and particle separation (removing all regions with 20 or less contagious white pixels thus eliminating light reflections).

\subsection{Descriptive variables and statistical analyses}

The analysis of the preprocessed images was also performed with SEMPER 9. We obtained radial extension of the mycelia by measuring the distance between inoculum and foraging front along eight lines (angle between lines $45^{\circ}$ ) and calculating the average. Radial extension is easy to measure, but not easily interpreted if observed in isolation: rapid extension can be interpreted as indicative of a vigorous, fast-growing mycelium; however, it could also be interpreted as the opposite indicating an escape response from unfavourable conditions (Boddy, 1999).

Hyphal cover was determined by counting the white pixels making up a captured image. Hyphal cover is broadly equivalent to mycelial biomass since they can be converted into each other by weighing mycelia of determined cover and obtaining calibration curves.
We characterised the space-filling properties of the mycelia by determining their fractal properties. Fungal mycelia show self-similarity over a range of scale and thus can be approximated to fractals as has been shown in numerous studies (e.g. Ritz and Crawford, 1990; Bolton and Boddy, 1993; Jones et al., 1993; Senesi and Boddy, 2002). Fractal geometry has turned out to be an extremely useful means of quantifying the extent to which mycelia permeate space in relation to the extent of the system (Boddy, 1999; Boddy et al., 1999); its application permitted the identification of small changes which could not be detected by biomass measurement (Donnelly and Boddy, 1998). We measured fractal dimensions of mycelia by the box-count method (Hastings and Sugihara, 1993): we overlaid each image electronically with grids of square boxes of size 361 pixels and recorded the number of boxes intersecting white pixels (representing fungal hyphae) (Donnelly et al., 1995). For a series of boxes with varying side length $s$, the number of boxes intersected, $N$, is related to $s$ according to

$N(s)=C \times s^{-D_{\mathrm{B}}}$

with $C$ indicating a constant and $D_{\mathrm{B}}$ indicating the boxcount dimension of the mycelium. Subsequently, we obtained $D_{\mathrm{B}}$ by a linear regression of the linear portion of $\log N(s)$ against $\log s$. The lower limit of resolution is determined by the pixel size; according to Markx and Davey (1990); Obert et al. (1990) the upper limit is usually determined by $25 \%$ of the maximum width of the image set.

When all intersected boxes are used for the calculation of $D$ from Eq. (3), $D_{\mathrm{B}}$ is called the mass fractal dimension $D_{\mathrm{BM}}$. For estimation of the surface fractal dimension $D_{\mathrm{BS}}$, the boxes that contain white pixels only are omitted from calculation. The distinction between mass and surface dimension allows for the distinction between mycelia that are fractal only at their margins having plane-filled interiors ('border fractal'), and those where the interior of the system has lacunae ('mass fractal') (Obert et al., 1990). Since border boxes are not entirely filled by the mycelium, we applied a corrected formula (Eq. (4)) to avoid an overestimate of the area of the structure at large box sizes (Kaye, 1989)

$N(s)-1 / 2 N_{\text {border }}(s)=C \times s^{-D_{\mathrm{BS}}}$

with $N_{\text {border }}$ indicating the boxes intersected by the margin of the mycelium.

In addition to the use of the descriptors mycelial extension, hyphal cover, and the fractal dimensions $D_{\mathrm{BM}}$ and $D_{\mathrm{BS}}$, we carefully observed the model systems visually to detect qualitative features of mycelial growth patterns.

The application of a two-factorial ANOVA (time $\times$ treatment) was not possible due to heteroscedasticity of the data; since log transformation did not yield homogenous variances we applied a series of one-way ANOVAs for the treatment factor $(P<0.05)$, subsequently testing for differences between single treatments by Tukey's honest 
significant difference (HSD). Qualitative features were registered on a binary base (present/absent) and treatment differences compared by contigency tables.

\section{Results}

\subsection{Quantitative descriptors}

The grazing intensity experiment lasted 47 days (Fig. 1). Mycelial extension and hyphal cover increased continually in the controls whereas from $26 \mathrm{~d}$ the grazed mycelia lagged significantly behind the control $(P<0.05$ for all HSD between control and systems with 40 and 60 $F$. quadrioculata from $26 \mathrm{~d}, P<0.05$ for all HSD between control and systems with $20 \mathrm{~F}$. quadrioculata from $35 \mathrm{~d}$ ). No increase in mycelial extension and hyphal cover was detectable in the treatments with $60 F$. candida from day 30 onwards. Generally, both mycelial extension and hyphal cover decreased with increasing grazing density. At $47 \mathrm{~d}$ the controls were significantly higher for both variables than all the other treatments $(P<0.01$ for all HSD between control and any treatment). Mycelial extension in the $20 \mathrm{~F}$. candida treatment was higher (HSD, $P<0.01$ ) than in the 60 $F$. candida treatment, however, no differences were detectable for hyphal cover between the grazed treatments (all HSD $P>0.05$ ).

The fractal descriptors showed different behaviour (Fig. 1): while $D_{\mathrm{BM}}$ was not distinguishable between treatments throughout the experiment (except at $35 \mathrm{~d}$ when $D_{\mathrm{BM}}$ was higher in the control), $D_{\mathrm{BS}}$ was consistently higher in the controls from $30 \mathrm{~d}$ when $D_{\mathrm{BS}}$ reached a constant value. Like mycelial extension and hyphal cover, $D_{\mathrm{BS}}$ decreased monotonously with grazing intensity. At $47 \mathrm{~d}$ the control differed significantly from the 40 and 60 $F$. candida treatments (HSD, $P<0.01$ and $P<0.05$,
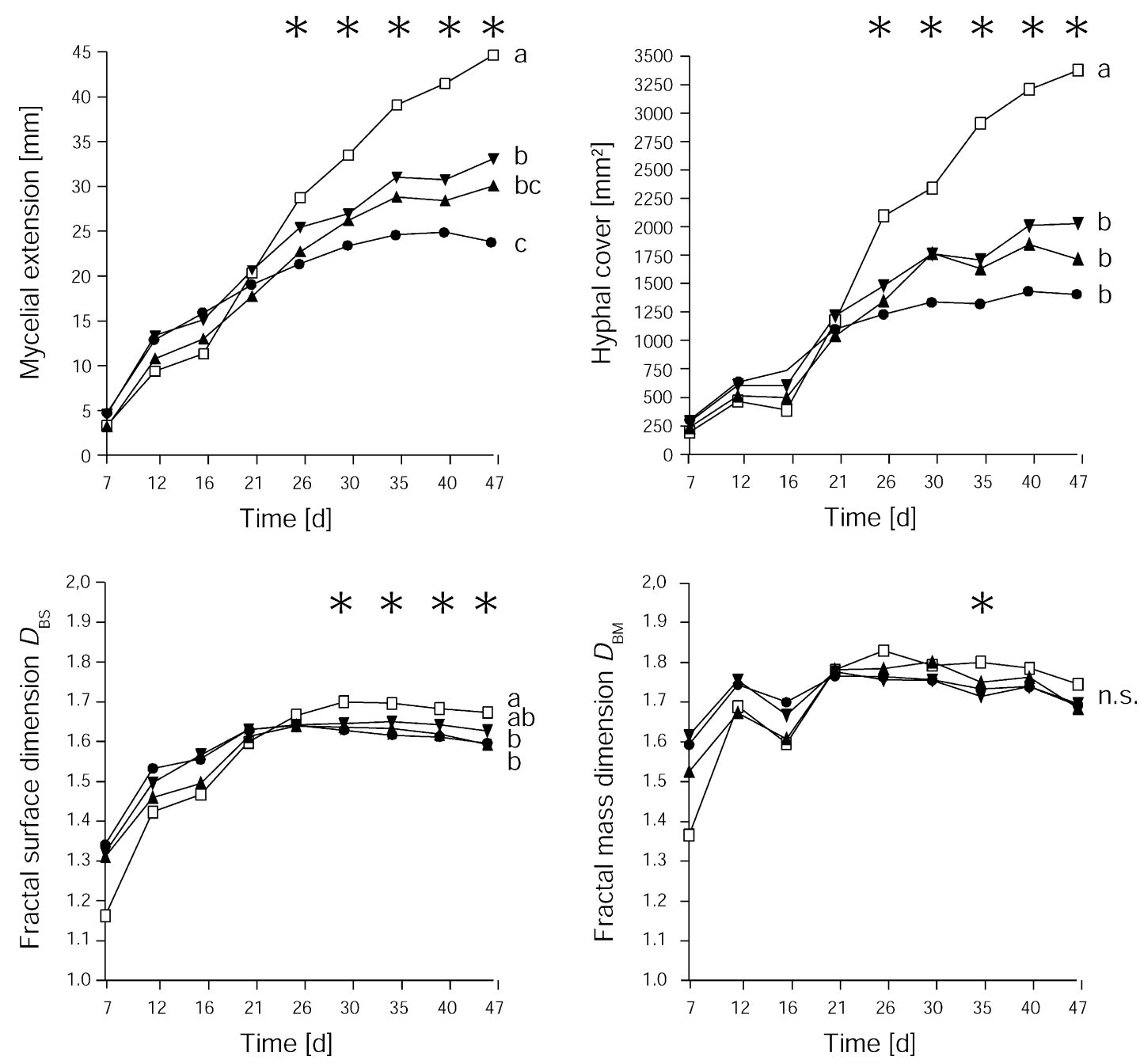

Fig. 1. Effects of different grazing densities of the collembolan $F$. candida on mycelial extension, hyphal cover, the fractal surface dimension, $D_{\mathrm{BS}}$, and the fractal mass dimension, $D_{\mathrm{BM}}$, of the fungus $H$. fasciculare. $\square$, control; $\mathbf{\nabla}, 20 ; \boldsymbol{\Lambda}, 40 ; \bullet, 60$ individuals per system. Asterisks indicate days with statistically significant differences between treatments; treatments sharing letters to the right of each diagram are not significantly different $(P>0.05)$ at day 47. 
respectively) whereas the treatment with $20 \mathrm{~F}$. candida could not be distinguished (HSD, $P>0.05$ ) from both the control or the other treatments.

Since the batch of $H$. fasciculare used for the experiment on response to grazing by different species appeared to grow more vigorously, the experiment ran for only $42 \mathrm{~d}$ (Fig. 2). As in the grazing intensity experiment, the control showed the largest increase in mycelial extension and hyphal cover, although not being significantly different (HSD, $P>0.05$ ) from the treatments with $P$. minuta, $H$. tullbergi and the species-mix during $42 \mathrm{~d}$. As observed in the $20 \mathrm{~F}$. candida treatment of the grazing intensity experiment, $F$. candida efficiently decreased the rate of mycelial extension: in the $F$. candida treatment hyphal cover was significantly lower than in the control (HSD, $P<0.001$ ) and the treatments with $P$. minuta (HSD, $P<0.01$ ) and $H$. tullbergi(HSD, $P<0.05)$ by $42 \mathrm{~d}$; the species mix showed intermediate values and was not statistically distinguishable (HSD, $P>0.05)$ from the other treatments. The fractal descriptors behaved similarly over time as in the grazing intensity experiment, but did not show any differences (all ANOVAs $P>0.05)$ between the treatments.

\subsection{Qualitative observations}

A considerable proportion of systems showed a remarkable switch in fungal foraging pattern during the experiments: While some mycelia maintained their initial growth pattern with a broad contiguous foraging front and uniform growth in all directions, some mycelia developed fast growing sectors while other sectors stopped growth completely, leading to narrow and increasingly independent search fronts (Fig. 3). Since observations were too few to test for the effect of single experiments or
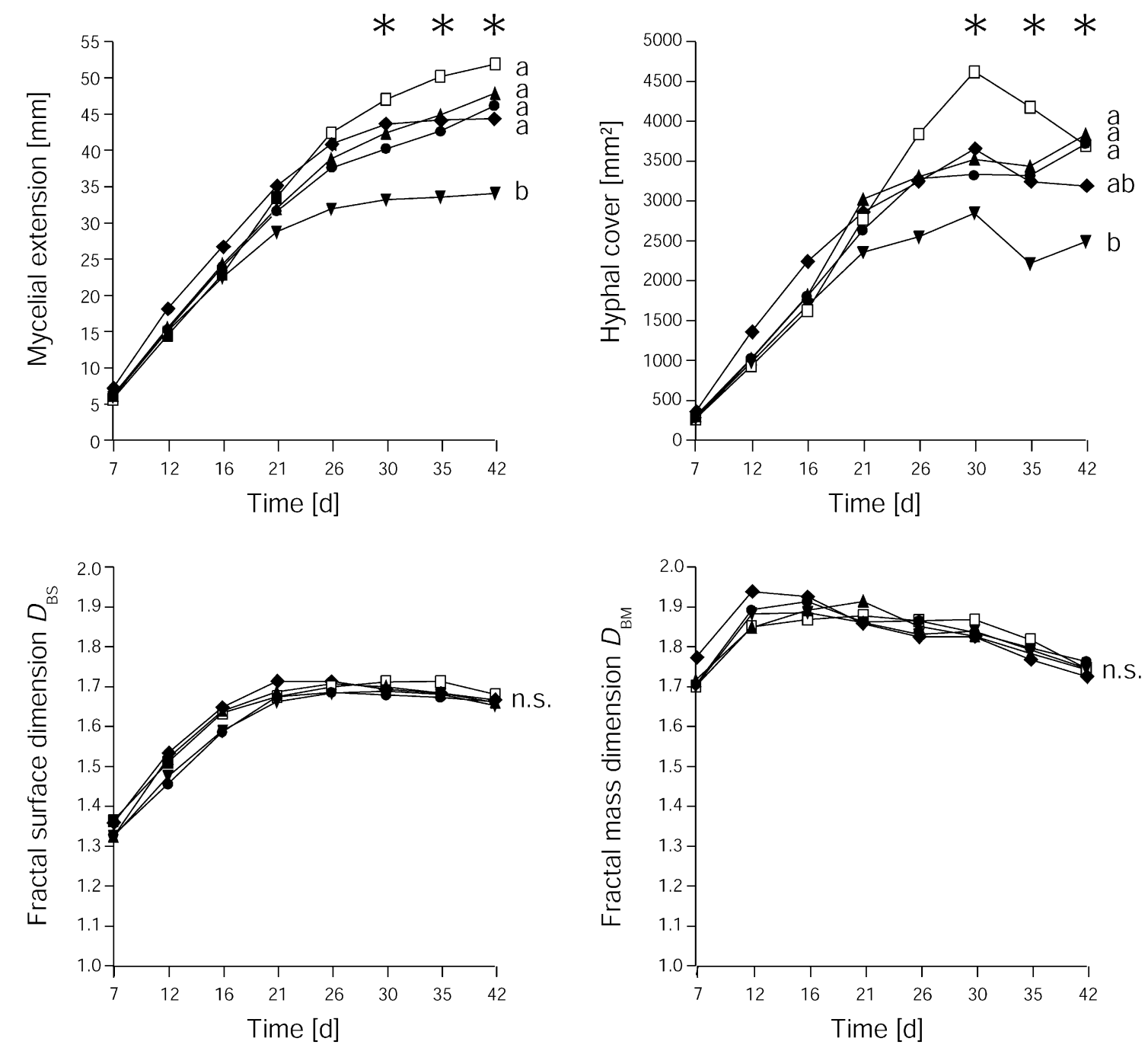

Fig. 2. Effects of different collembolan species on mycelial extension, hyphal cover, the fractal surface dimension, $D_{\mathrm{BS}}$, and the fractal mass dimension, $D_{\mathrm{BM}}$, of the fungus $H$. fasciculare. $\square$, control; $\boldsymbol{\nabla}, 20$ individuals of $F$. candida; $\boldsymbol{\Lambda}, 50$ P. minuta; $\bullet, 40$ H. tullbergi; $\bullet$, a species mix of 7 F. candida, 17 P. minuta and $13 \mathrm{H}$. tullbergi per system. Asterisks indicate days with statistically significant differences between treatments; treatments sharing letters to the right of each diagram are not significantly different $(P>0.05)$ at day 42. 


\section{Ungrazed}
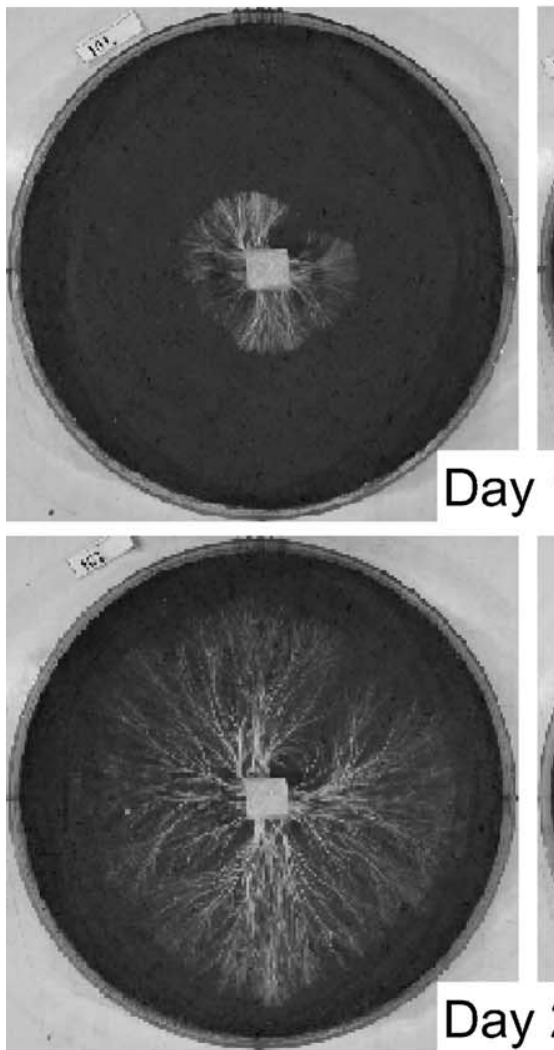

\section{Day 26}

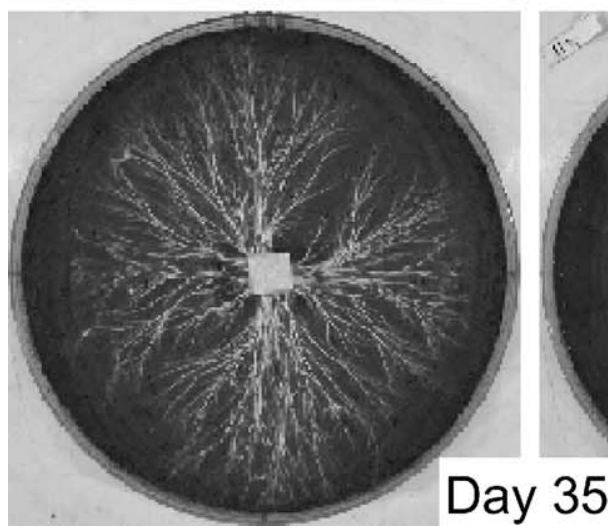

Grazed
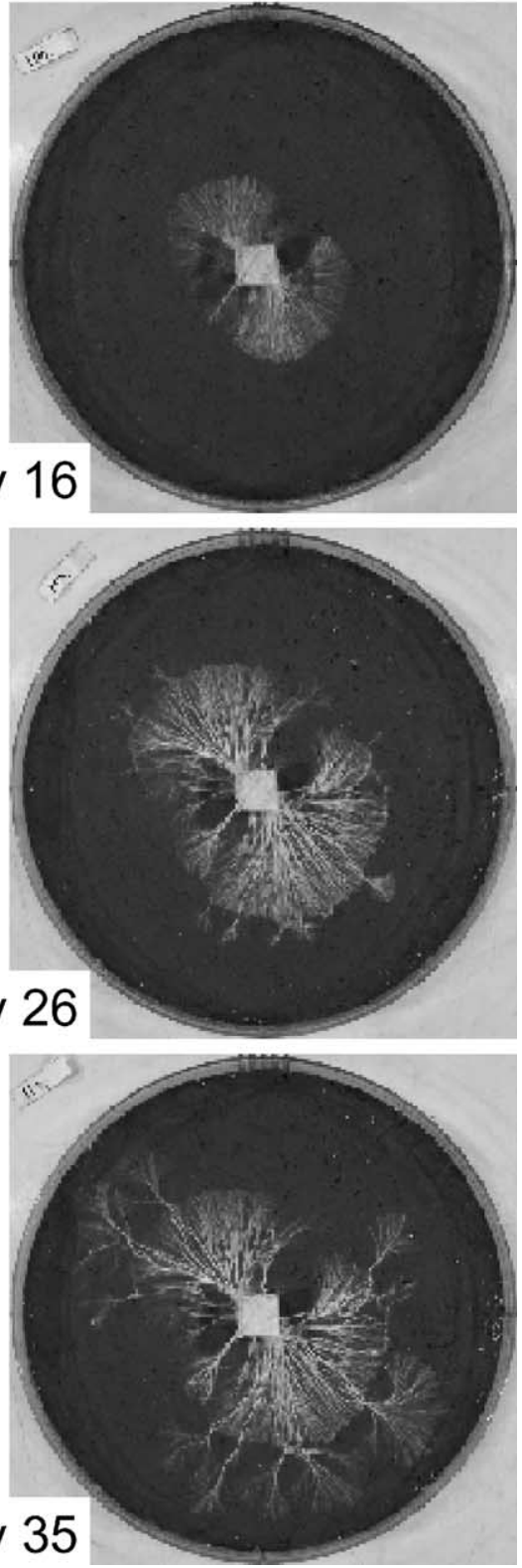

Fig. 3. Temporal development of an ungrazed mycelium of $H$. fasciculare maintaining 'phalanx' growth-strategy (left) and of a mycelium grazed by collembolans switching to 'guerilla' growth-strategy (right) at days 16 (the day collembolans were added), 26 and 35.

Table 1

Frequency of grazed and ungrazed systems containing a mycelium that switched or maintained its growth pattern

\begin{tabular}{lll}
\hline Statistic & & Maintained growth-pattern \\
\hline $2 \times 2$-contingency table & Ungrazed mycelia & 20 \\
& Grazed mycelia & 50 \\
Post-hoc probabilites & Ungrazed mycelia & 0 \\
& Grazed mycelia & 0.58 \\
\hline
\end{tabular}

The contingency table was used for calculating Fisher's exact test. The post-hoc probabilities that a system containing a maintained or switched growthpattern were grazed or ungrazed were calculated according to the special case of Bayes' theorem for equal a priori probabilities $(P=0.5)$ for two alternative hypotheses, $P\left(\mathrm{~A}_{i} \mid \mathrm{B}\right)=P\left(\mathrm{~B} \mid \mathrm{A}_{i}\right) /\left[P\left(\mathrm{~B} \mid \mathrm{A}_{1}\right)+P\left(\mathrm{~B} \mid \mathrm{A}_{2}\right)\right]$, with $\mathrm{A}_{i}$ indicating hypothesis $\mathrm{A}_{1}$ or $\mathrm{A}_{2}$. 


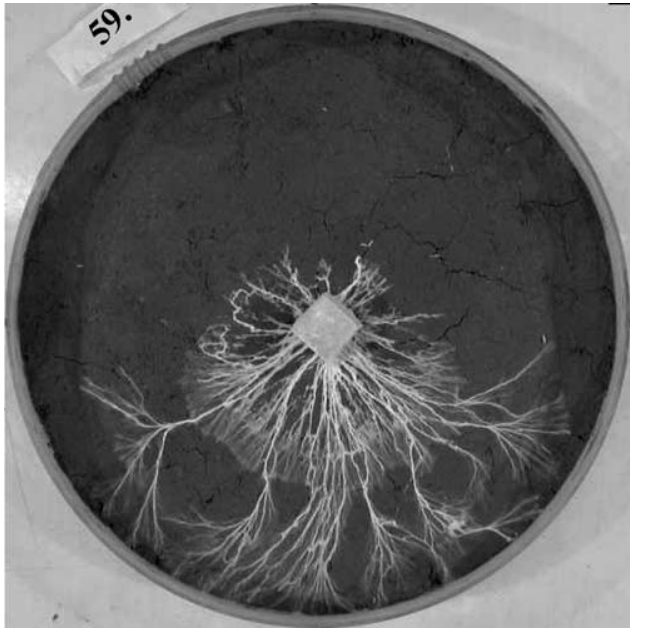

Fig. 4. A mycelium of $H$. fasciculare forming anastomoses, visible by a faint white circle behind the foraging front.

treatments, we lumped all observations $(n=89)$ into 'grazed' and 'ungrazed' systems. Although mycelia with a stable growth pattern were found in grazed as well as in ungrazed systems, switching of growth strategy was found in grazed systems only (Table 1). This pattern was significantly different from a random pattern (Fisher's two-tailed exact test, $P=0.021$ ). Expressed from a Bayesian point of view, the post-hoc probability $P$ (switched|grazed) that a switched mycelium will be the result of grazing is 1.00 , the post-hoc probability $P$ (switchedlungrazed) that it will have occurred in an ungrazed system is 0.00 (Table 1).

About $10 \%$ of the mycelial systems developed tangential anastomoses behind the foraging front, thus forming cross connections between radial hyphae, visible as nearly concentric circles (Fig. 4). However, there was no significant difference in their frequency between grazed and ungrazed systems (Fisher's two-tailed exact test, $P=0.58$; all Bayesian post-hoc probabilities were lying between 0.48 and 0.53 ).

\section{Discussion}

\subsection{Effects of grazing intensity}

Mycelial extension and hyphal cover monotonously decreased with increased grazing intensity, indicating a linear relationship between grazing intensity and fungal growth at least for the range of collembolan density used. The highest density used (60 individuals of $F$. candida per system) corresponded to a density of 38,500 individuals $\mathrm{m}^{-2}$ which lies well within the range of collembolan densities observed in the field (Petersen and Luxton, 1982). No stimulation or acceleration of fungal growth, as reported by van der Drift and Jansen (1977); Bengtsson and Rundgren (1983) or Hedlund et al. (1991), was observed.
However, as in the experiments of Hanlon and Anderson (1979) grazing by $F$. candida always led to reduction in fungal biomass (measured in the present paper as hyphal cover), regardless of grazing intensity.

Surface fractal dimension, $D_{\mathrm{BS}}$ decreased at higher grazing intensities (40 and $60 F$. candida per system) indicating a less branched foraging front due to the regression of fine hyphae and the development of mycelial cords. This might be either a direct effect of the consumption of fine hyphae by the collembola, or an indirect growth response of the fungus. Mass fractal dimension, $D_{\mathrm{BM}}$, did not respond significantly to grazing; however, throughout the experiment values of $D_{\mathrm{BM}}$ were highest in the control, indicating that space filling properties of the mycelia were also affected.

\subsection{Species specific effects}

Although all species used in the experiments have chewing mouth-parts, their effects on fungal growth were remarkably different: whereas $20 \mathrm{~F}$. candida decreased mycelial extension and fungal cover distinctly, yielding similar results to the corresponding treatment of the grazing intensity experiment, neither $P$. minuta nor $H$. tullbergi exerted a similar effect upon $H$. fasciculare. Obviously, grazing by smaller organisms-although performed by a larger number of individuals - can be compensated for by the fungus whereas the damage by larger grazers alters fungal growth. These size-dependent effects might in part explain the different outcomes (sometimes increase, sometimes decrease in biomass) of experiments using collembolans as grazers of fungi (van der Drift and Jansen, 1977; Bengtsson and Rundgren, 1983; Hedlund et al., 1991; Hanlon and Anderson, 1979). Species-specific effects have also been described with regard to $N$-mineralisation (Mebes and Filser, 1998), the latter occurring as a result of grazing on fungi. Thus, although often lumped to a single functional group-'fungivorous collembola' or even 'fungivorous microarthropods' - in soil ecological studies (e.g. Brussaard, 1998), we should be very cautious about overgeneralisation of the role of collembolans in interactions with soil fungi. The species mix resulted in intermediate values of mycelial extension and hyphal cover, suggesting an additive effect of the component species in the mixture.

The decrease of hyphal cover in the control from $30 \mathrm{~d}$ is due to increased formation of cords in ageing mycelia. This effect has been observed in various experiments with $H$. fasciculare in the absence of grazing (Donnelly et al., 1995; Boddy et al., 1999). Due to the higher vigour of the batch of the fungus used in the specific response experiment, the stage of cord development appeared earlier (day 30, hyphal cover $4600 \mathrm{~mm}^{2}$ ) than in the grazing density experiment where it could not be observed (hyphal cover on day 47: $3400 \mathrm{~mm}^{2}$ ).

$D_{\mathrm{BS}}$ had higher values than $D_{\mathrm{BM}}$ throughout both experiments as in other studies (e.g. Boddy, 1999) indicating 
surface-fractal growth: the dense space-filling mycelium with a distinctly defined foraging front is particularly successful in colonising small local and relatively homogenously dispersed resources (e.g. Boddy, 1999); it corresponds to the phalanx strategy sensu Schmid and Harper (1985) and is characteristic of fungi exploiting resources. Fractal dimensions did not show any effect in the specific response experiment. This resembles the results obtained in the grazing intensity experiment, where low numbers of $F$. candida did not affect $D_{\mathrm{BS}}$ and $D_{\mathrm{BM}}$. After an initial increase, the fractal mass dimension $D_{\mathrm{BM}}$ decreased during the experiment, indicating that spaces were opening within the mycelium, as also reported in other studies (e.g. Boddy, 1999). Under nutrient-limited conditions the decrease of the fractal dimensions is interpretable as a strategy of biomass conservation: the nutrients set free by the autolysis of finer hyphae are absorbed, translocated within the mycelium and invested in mycelial growth at the foraging front (Bolton and Boddy, 1993). The lack of decrease in $D_{B M}$ in the density experiment may reflect the less vigorous development of the colony, and might have been expected to occur later if the experiment had continued.

\subsection{Switch in growth strategy}

The changes of growth patterns of $H$. fasciculare indicate a change in foraging strategy: the switch from phalanx to guerilla strategy (Schmid and Harper, 1985). The guerilla strategy is most effective for foraging for large and heterogenously dispersed high-quality resources of larger areas. Phalanx and guerilla strategy, thus, can also be interpreted as exploitative and explorative growth.

The switch from phalanx to guerilla strategy never happened in ungrazed systems; the resources provided by the model system seem to be sufficient to permit an exploitative growth-pattern, at least over the first weeks of the experiment. $38 \%$ of the grazed mycelia, however, did not maintain their initial growth-pattern and changed to a longer ranged explorative strategy. As in the experiment of Hedlund et al. (1991) we interpret this observation as a fugitive response and as a strategy for quickly escaping from places where grazing pressure is experienced. Here the guerilla strategy does not resemble an exploration for nutrients or energy but an exploration for enemy-free space, for pores and substrates out of reach of the grazer. Moreover, the thicker cords produced during explorative growth provide more efficient protection from grazing: many mycelial cords consist of an outer rind, comprising a layer of hyphae of similar diameter and often encrusted with crystals (Boddy, 1993).

The switching response by $H$. fasciculare was not only observed in $40 \%$ of the systems with $F$. candida (the species that is able to affect growth considerably as shown in both experiments) and in $29 \%$ of the mixed treatment (also containing $F$. candida), but also in systems grazed by P. minuta (50\%) and H. tullbergi (29\%). Although the smaller species did not affect rate of mycelial extension or fungal cover, they did influence fungal growth strategy. In these treatments the loss of fine hyphae is most probably not due to collembolan grazing - as it could be the case in treatments with $F$. candida-but to the translocation of internal reserves of energy and nutrients to the narrowfronted extending sectors of the mycelia of $H$. fasciculare.

\subsection{Conclusions}

H. fasciculare responds to grazing density and to the specific composition of the grazing microarthropod assemblage in a complex way: it may suffer from damage caused by few but large collembolans, affecting extension as well as cover of the mycelium; it may compensate for the biomass loss caused by more but slightly smaller collembolans (the linear body-size between large and small species in our experients relates as only 1:0.69); it may respond by switching from exploitative phalangeal growth to an explorative guerilla-strategy most probably accompanied by internal reallocation of resources from the dense interior hyphal network behind the initial mycelial margin to narrow fast-expanding foraging fronts. At the moment we can neither estimate the longevity of these responses nor their spatial shape and extension in more complex substrates than on the surface of compressed soil. Hedlund et al. (1991) have shown that growth responses of fungi to grazing can be reversible; having reached enemy-free refuges $H$. fasciculare may well switch back to an exploitative mode of growth. However, testing this hypothesis and determining potential quantity and distance of resource translocation within a mycelium requires larger and longer-term experimental systems. This will be indispensable for getting a first insight into the spatial aspects of microarthropod-fungal interactions and their consequences for the transport of energy and nutrients in forest soils.

\section{Acknowledgements}

This study was funded by the Deutsche Forschungsgemeinschaft (Project 'Der Einfluss der Beweidung durch Kleinarthropoden auf Wachstumstrategien holzabbauender Pilze').

\section{References}

Bengtsson, G., Rundgren, S., 1983. Respiration and growth of a fungus Morteriella isabellina, in response to grazing by Onychiurus armatus (Collembola). Soil Biology and Biochemistry 15, 469-473.

Boddy, L., 1993. Saprotrophic cord-forming fungi: warfare strategies and other ecological aspects. Mycological Research 97, 641-655.

Boddy, L., 1999. Saprotrophic cord-forming fungi: meeting the challenge of heterogeneous environments. Mycologia 91, 13-32.

Boddy, L., 2000. Interspecific combative interactions between wooddecaying basidiomycetes. Federal European Microbiological Society Microbial Ecology 31, 185-194. 
Boddy, L., Wells, J.M., Culshaw, C., Donnelly, D.P., 1999. Fractal analysis in studies of mycelium in soil. Geoderma 88, 301-328.

Bolton, R.G., Boddy, L., 1993. Characterization of the spatial aspects of foraging mycelial cord systems using fractal geometry. Mycological Research 97, 762-768.

Booth, R.G., Anderson, J.M., 1979. The influence of fungal food quality on the growth and fecundity of Folsomia candida. Oecologia 38, 317-323.

Brussaard, L., 1998. Soil fauna, guilds, functional groups and ecosystem processes. Applied Soil Ecology 9, 123-136.

Donnelly, D.P., Boddy, L., 1997. Development of mycelial systems of Stropharia caerulea and Phanerochaete velutina on soil: effect of temperature and water potential. Mycological Research 101, 705-713.

Donnelly, D.P., Boddy, L., 1998. Developmental and morphological responses of myelial systems of Stropharia caerulea and Phanerochaete velutina to soil nutrient enrichment. New Phytologist 138, 519-531.

Donnelly, D.P., Wilkins, M.F., Boddy, L., 1995. An integrated image analysis approach for determining biomass, radial extent and box-count fractal dimension of macroscopic mycelial systems. Binary 7, 19-28.

Dunger, W., Fiedler, H.J., 1997. Methoden der Bodenbiologie. Gustav Fischer, Stuttgart, New York, 432p.

Edwards, C.A., 1967. Relationships between weights, volumes and numbers of soil animals. In: Graff, O., Satchell, J.E. (Eds.), Progress in Soil Biology, North Holland Publishing Company, Amsterdam, pp. 585-594.

Hanlon, R.D.G., Anderson, J.M., 1979. The effect of Collembola grazing on microbial activity in decomposition of leaf litter. Oecologia 38, 93-99.

Hastings, H.M., Sugihara, G., 1993. Fractals. A User's Guide for the Natural Sciences. Academic Press, London, 235p.

Hedlund, K., Boddy, L., Preston, C.M., 1991. Mycelial responses of the fungus, Morteriella isabellina, to grazing by Onychiurus armatus (Collembola). Soil Biology and Biochemistry 23, 361-366.

Jones, C.L., Lonergan, G.T., Mainwaring, D.E., 1993. A rapid method for the fractal analysis of fungal colony growth using image processing. Binary 5, 171-180.

Kaye, B.H., 1989. Image analysis techniques for characterising fractal structure. In: Avnir, D., (Ed.), The Fractal Approach to Heterogenous Chemistry-Surfaces, Collids, Polymers, Wiley, Chichester, pp. 55-66.

Leonard, M.J., Anderson, J.M., 1991. Grazing interactions between a collembolan and fungi in a leaf litter matrix. Pedobiologia 35, 239-246.

Markx, G.H., Davey, C.L., 1990. Applications of fractal geometry. Binary $2,169-175$
Mebes, K.-H., Filser, J., 1998. Does the species composition of Collembola affect nitrogen turnover? Applied Soil Ecology 9, 241-248.

Moore, J.C., St. John, T.V., Coleman, D.C., 1985. Ingestion of vesiculararbuscular mycorrhizal hyphae and spores by soil microarthropods. Ecology 66, 302-312.

Obert, M., Pfeifer, P., Sernetz, M., 1990. Microbial growth patterns described by fractal geometry. Journal of Bacteriology 172, $1180-1185$.

Petersen, H., Luxton, M., 1982. A comparative analysis of soil fauna populations and their role in decomposition processes. Oikos 39, 287-388.

Ritz, L., Crawford, J., 1990. Quantification of the fractal nature of colonies of Trichoderma viride. Mycological Research 94, 1138-1141.

Schmid, B., Harper, J.L., 1985. Clonal growth in grassland perennials. I. Density and pattern-dependent competition between plants with different growth forms. Journal of Ecology 73, 793-808.

Senesi, N., Boddy, L., 2002. A fractal approach for interactions between soil particles and microorganisms. In: Huang, P.M., Bollag, J.-M., Senesi, N. (Eds.), Interactions Between Soil Particles and Microorganisms, Wiley, Chichester, pp. 41-83.

Smith, M.L., Bruhn, J.N., Anderson, J.B., 1992. The fungus Armillaria bulbosa is among the largest and oldest living organisms. Nature 356, $428-431$.

Thompson, W., Rayner, A.D.M., 1982. Structure and development of mycelial cord systems of Phanerochaete laevis in soils. Transactions of the British Mycological Society 78, 193-200.

van der Drift, J., Jansen, E., 1977. Grazing of springtails on hyphal mats and its influence on fungal growth and respiration. In: Lohm, U., Persson, T. (Eds.), Soil organisms as components of ecosystems, Ecological Bulletin, 25., pp. 203-209.

Varga, J., Naar, Z., Dobolyi, C., 2002. Selective feeding of collembolan species Tomocerus longicornis (Mull.) and Orchesella cincta (L.) on moss inhabiting fungi. Pedobiologia 46, 526-538.

Verhoef, H.A., de Goede, R.G.M., 1985. Effects of collembolan grazing on nitrogen dynamics in a coniferous forest. In: Fitter, A.H., Atkinson, D., Read, D.J., Usher, M.B. (Eds.), Ecological Interactions in Soil: Plants, Microbes and Animals, Blackwell, Oxford, pp. 367-376.

Wells, J.M., Harris, M.J., Boddy, L., 1998. Encounter with new resources causes polarised growth of the cord-forming basidiomycete Phanerochaete velutina. Microbial Ecology 36, 372-382. 